\title{
Nuchal Fibroma: An Uncommon Neck Mass
}

\author{
Soumyajit Das, ${ }^{1}$ Subhasish Mukherjee, ${ }^{2}$ Barun Sharma, ${ }^{3}$ Subash Tamang ${ }^{1}$
}

\section{Introduction:}

\section{$\underline{\text { ABSTRACT }}$}

Nuchal fibroma or collagenosis nuchae is a rare benign tumour. It is a slow growing neoplasia of unknown etiogenesis, asymptomatic and of variegated histology. They are more common in males but our case was a female patient. The presentation may mimic sarcoma at times.

\section{Case Report}

An unusual case of a very large neck mass in a 62 years old female patient is reported. The growth involved the dorsocervical region over a period of approximately 8 years but remained asymptomatic. The mass was excised and post excision histopathology was reported as nuchal fibroma.

Discussion

The case reported is large compared to the usual size of nuchal fibroma. Association with diabetes mellitus and Gardner's syndrome has been reported in literature. MRI is the imaging of choice to establish the differential diagnosis.

Kevwords:

Head and Neck Neoplasms; Fibroma; Neck

$\mathrm{N}$ uchal fibroma is a rare benign entity. This was first described in 1988 by Enzinger and Weiss. ${ }^{1}$ It consists of a subcutaneous and dermal proliferation of collagen tissue with hypocellularity, entrapped adipocytes, lack of elastin tissue with interspersed nerve fibers. ${ }^{1,2}$ This arises mostly in the dorso-cervical area with predilection for interscapular and paraspinal regions..$^{1,2}$ The nomenclature has recently undergone a change from nuchal fibroma to nuchal type fibroma as it was observed to occur at anatomic sites other than the neck region. The age group affected generally ranges from 25 to 60 years but occurrence in younger age group is also known. ${ }^{1,2}$ The condition has been found to be associated with Diabetes Mellitus and Gardner's syndrome. ${ }^{2}$ Apart from the nuchal region, the other sites involved are the trunk area, forearm and the

1 - Sikkim Manipal Institute of Medical Sciences, Gangtok

2 - IQ City Medical College, Durgapur

3 - North Eastern Indira Gandhi Regional Institute of

Health and Medical Sciences (NEIGRIHMS), Shillong

Corresponding author:

Dr Soumyajit Das

email: drsoumya_entamch@rediffmail.com knee. ${ }^{3}$ Till date, total excision remains the treatment.

\section{Case Report}

A 62 year old female presented with a history of swelling in the right side of neck for the last six years. The swelling was painless and gradually progressive without any symptoms of neurological deficit. There was no history of sudden increase in size of the swelling or any respiratory or breathing difficulty. On examination a firm non-tender swelling of approximately $10 \mathrm{~cm} \mathrm{X} 8$ $\mathrm{cm}$ was noted in the right side of neck extending from the mastoid process to the upper border of clavicle. There was no fixity to the underlying structures or the overlying skin. (Fig. 1)

Ultrasonography of the neck swelling revealed a well-defined encapsulated mass lesion with internal heterogeneous echotexture along with posterior shadowing in right posterior triangle. Contrast enhanced computed tomography (CECT) of neck swelling revealed a well-encapsulated hypodense mass lesion measuring $10.9 \mathrm{~cm} \times 10.8 \mathrm{~cm} \times 12.2 \mathrm{~cm}$ in right posterior triangle with surrounding intact fat plane, thin capsule and showing heterogeneous internal density of $20 \mathrm{HU}-55 \mathrm{HU}$ 


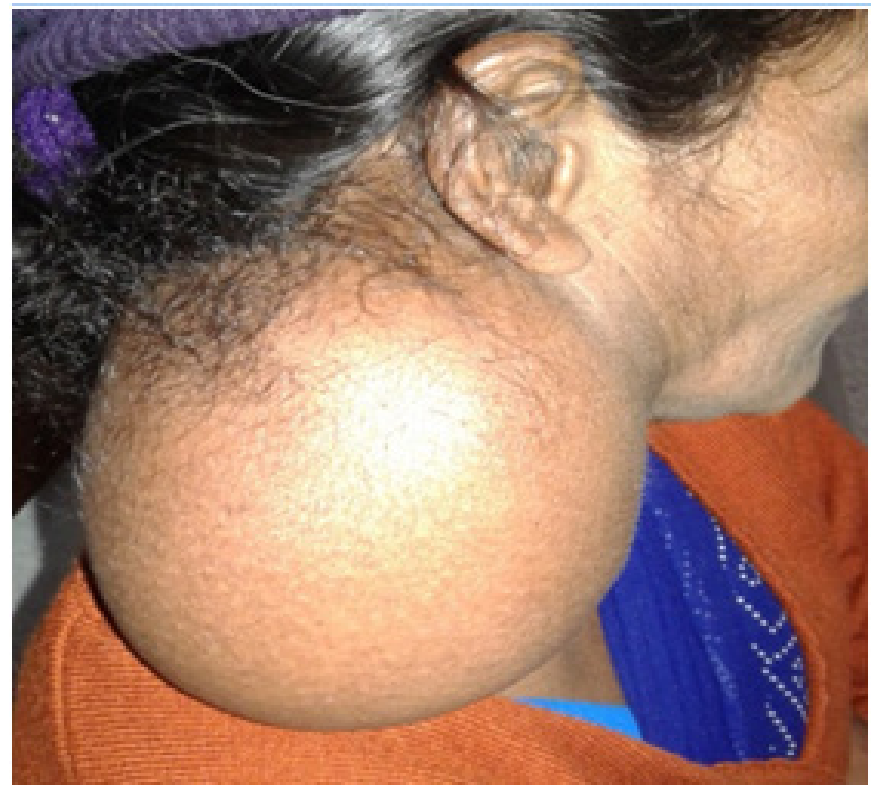

Fig. 1. Clinical photograph of the swelling in the neck

and without any significant contrast enhancement. The mass was displacing the sternocleidomastoid muscle and parotid gland anteriorly, trapezius muscle posteriorly and levator scapulae muscle medially. MRI of neck swelling revealed $\mathrm{T} 1$ and T2 heterogeneity with whorl appearance and predominant $\mathrm{T} 1$ isointensity and T2 hyperintensity along with hypointensity in between. Areas of diffusion restriction seen without any evidence of GRE blooming (Fig. 2A-D)

The mass was excised by a transverse neck incision. Intraoperatively a well defined mass was found which showed minimal adherence to the trapezius muscle in the posterior aspect of the tumor. The cut surface of the tumor was pale and firm. Microscopic features included a hypocellular stroma with bundles of collagen (Fig 3).

During the follow up visit the patient was evaluated for colonic polyposis by colonoscopy. The patient was not diabetic and there was no evidence of polyposis of the colon. Her family history was unremarkable. The clinical features were unremarkable for associated Gardner's syndrome.

\section{Discussion}

Nuchal type fibroma as described by Enzinger and Weiss

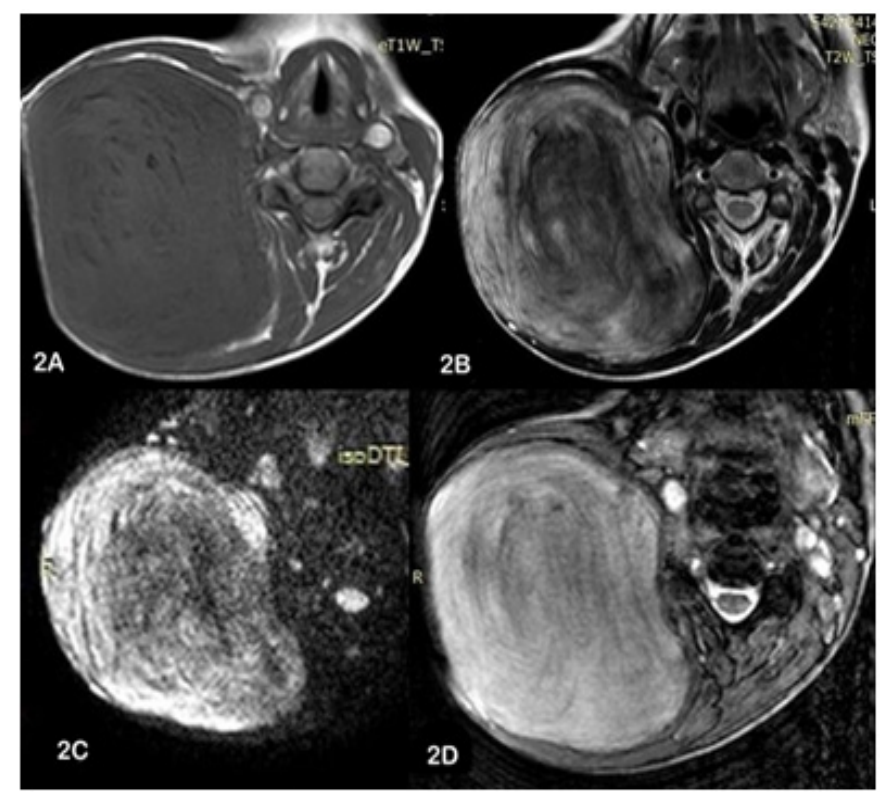

Fig. 2. Magnetic Resonance Imaging (MRI) showing heterogeneous signal intensities with whorl appearances in $\mathrm{T} 1$ weighted (2A) and $\mathrm{T} 2$ weighted (2B) images with areas of diffusion restriction (2C) without any GRE blooming

(2D).

in 1988 is a solitary, non encapsulated, subcutaneous swelling usually located on the back of the neck or dorsum 1 . They are slow growing asymptomatic tumours of unknown etiopathogenesis. It is more commonly seen in males in the age group of 25 to 60 years but the limits may stretch on either side. Mostly the size recorded till date is between $2 \mathrm{~cm}$ to $8 \mathrm{~cm}$ but in our case the tumour measured approximately $13.5 \mathrm{~cm}$ by $10 \mathrm{~cm}$. Association with diabetes mellitus and Gardner's syndrome has been seen in many cases. However, in some cases a spectrum of lesions consisting of osteomas, various soft tissue tumours and adenomatous polypii of the colon has led to the belief that this maybe a sentinel condition for Gardners' syndrome. ${ }^{4}$ In majority of cases nuchal type fibromas are solitary lesions but multiple tumours have also been reported.

The pathogenesis of nuchal type fibroma till date has not been established but co morbidities like diabetes mellitus and Gardner's syndrome are documented. There may be genetic predisposition specially in patients with Gardner's syndrome. Repeated trauma has recently been claimed as a possible etiopathogenetic factor. ${ }^{5}$ The 


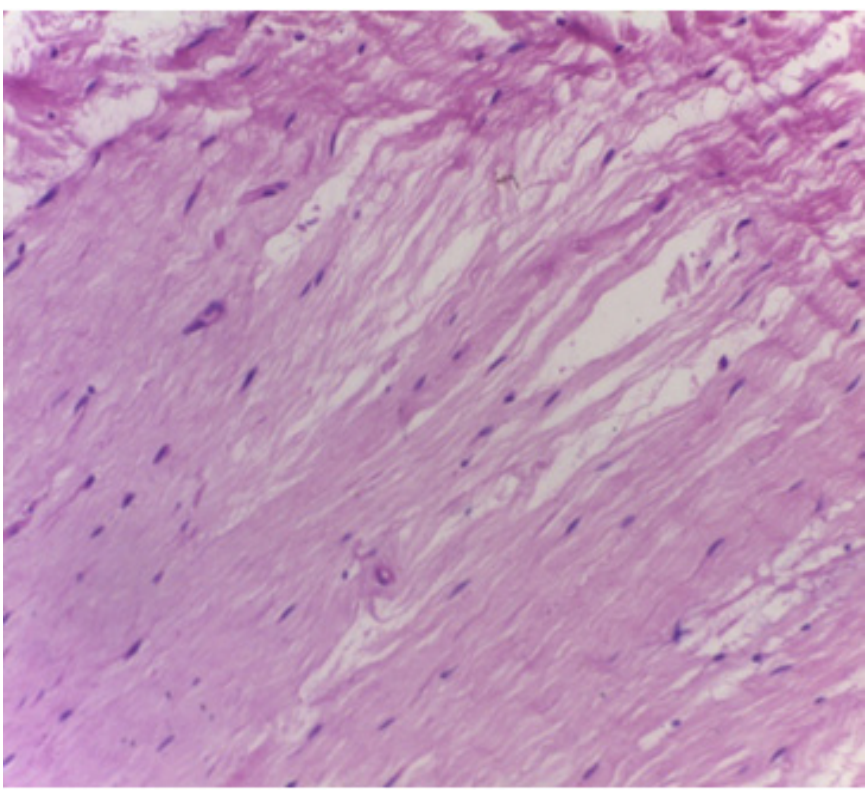

Fig. 3. Microscopic features of the tumor showing bundles of collagen tissue with hypocellular stroma (H\&E, 10X) present case did not have any other co morbidity. The macroscopic appearance of the tumor may appear tan to greyish lobulated mass and tan to white cut margins which may be irregular and mimick sarcoma. ${ }^{6}$

Histopathologically nuchal type fibroma consists of haphazardly arranged collagen fibers with scattered fibroblasts and entrapped adipose tissue, nerve fibers and adenexal structures. ${ }^{7}$ Histopathologically, differential diagnosis includes fibrolipoma - an encapsulated tumour unlike nuchal type fibroma. Collagenous and desmoid fibromas are ruled out by positive CD34 expression and negative Betacatenin reaction. Dermatofibroma is differentiated as the epidermis usually has pseudoepitheliomatous hyperplasia and hypertrophied basal cell. ${ }^{5}$

MRI is the imaging of choice to establish the differential diagnosis. Nuchal type fibromas may appear as ill defined low signal intensity lesions in T1 and T2 weighted images. ${ }^{5}$

Complete excision is the treatment of choice. This tumor has a tendency to recur. ${ }^{2}$ In the present case there was no recurrence at six month follow up.

\section{Conclusion}

Nuchal type fibroma of the cervical region are rare benign tumors and should be considered in the differential diagnosis of neck swellings. These tumors can cause symptoms due to the mechanical effect of enlarging size and at time may be associated with Gardner's syndrome and diabetes mellitus.

\section{Acknowledgement}

We would like to thank Dr Amlan Gupta, Professor and Head, Department of Pathology for providing us with the histological picture and his generous support.

\section{References}

1. Michal M, Fetsch JF, Hes O, Miettinen M. Nuchal- type fibroma: A clinicopathologic study of 52 cases. Cancer 1999; 85(1):156-63

2. Gong Y, Zhao X, Wu D, Liu J. Nuchal type fibroma of the shoulder ; A case report and review of literature. Oncol let. 2016; 11(6):4152-4

3. Shin JB, Son SW, Kim H. Nuchal type fibroma of the Coccyx. Ann Dermatol. 2008; 20 (1):41-4

4. Wehrli Bret M, Weiss Sharon W, Yandon Suzanne, Coffin Cheryl M. Gardner associated fibromas (GAF) in young patients : A distinct fibrous lesion that identifies unsuspected Gardner's syndrome and risk of fibromatosis. Am J Surg Pathol. 2001; 25(5):645-51

5. Lee CC, Lai CS, Lin CH, et al. Extra nuchal - type fibroma associated with repetitive blunt trauma during religious activities. Trauma case reports 2016; 4:16-20

6. Ramezani M, Masnadjam M, Sadeghi M. Extranuchal-type Fibroma Mimicking Low Grade Sarcoma: A Case Report and Review of the Literature. Am J Cancer Prev. 2017; 5(1):17-20 doi: 10.12691/ajcp-5-1-3

7. Do HunKim, Tae Han Kim, Nam Hee Sung, Hyoseung Shin, Ai Young Lee, Seung Ho Lee. Multiple nuchal type fibroma on the scalp : A case report. Ann Dermatol. 2015; 27(2):194-6. 